\title{
POINT OF COLLAPSE AND CONTINUATION METHODS FOR LARGE AC/DC SYSTEMS
}

\author{
Claudio A. Cañizares, Member \\ Escuela Politécnica Nacional-Quito \\ P.O. Box 17-08-8339, Quito, Ecuador
}

\author{
Fernando L. Alvarado, Senior Member \\ University of Wisconsin-Madison \\ Madison, Wisconsin 53706 USA
}

\begin{abstract}
This paper describes the implementation of both Point of Collapse (PoC) methods and continuation methods for the computation of voltage collapse poirts (saddle-node bifurcations) in large ac/dc systems. A comparison of the performance of these methods is presented for real systems of up to 2158 buses. The paper discusses computational details of the implementation of the PoC and continuation methods, and the unique challenges encountered due to the presence of high voltage direct current (HVDC) transmission, area interchange power control, regulating transformers, and voltage and reactive power limits. The characteristics of a robust $\mathrm{PoC}$ power flow program are presented, and its application to detection and solution of voltage stability problems is demonstrated.

Keywords: Voltage collapse, large ac/dc systems, saddlenode bifurcation, point of collapse, direct methods, continuation methods.
\end{abstract}

\section{INTRODUCTION}

During the last few years several methodologies for detecting saddle-node bifurcations in dynamic systems using steady state analysis techniques [1], have b'en tailored and applied to the determination of loadability limits of power systems. In this paper dynamic saddle-node bifurcations, or voltage collapse points, will be considered to be detectable by looking only for singularities of the steady state power flow Jacobian, since, under certain assumptions, saddlenode bifurcations of ac/dc dynamic systems with algebraic constraints can be shown to occur when the corresponding power flow Jacobian becomes singular $[6,7]$.

One simple alternative to find loadability limits is to use an ordinary power flow and to gradually increase loads until convergence is no longer obtained. In addition to the need for manual intervention, this approach often suffers from convergence difficulties and one is never certain where the limits actually are. A more precise determination of the proximity of a limit is essential when one is interested in effects of various possible control actions on the location of these limits. In addition, the conventional power flow method is generally not able to reliably find low voltage

92 WM 103-2 PWRS A paper recommended and approved by the IEEE Power System Engineering Committee of the IEEE Power Engineering Society for presentation at the IEEE/PES 1992 Winter Meeting, New York, New York, January $26-30,1992$. Manuscript submitted August 30, 1991; made available for printing December 31, 1991. solutions that are necessary for some of the direct energy function methods, or to directly determine the direction of maximum security increase. The Point of Collapse ( $\mathrm{PoC})$ method $[2,3,4,5]$ is one way of performing a direct computation of these limits. The method has been shown to be computationally feasible and well suited for determining proximity to voltage collapse in integrated ac/dc dynamic networks $[6,7]$. Continuation methods have also proven to be a good way of calculating bifurcation points in ac power systems $[5,8,9]$.

This paper presents brief quantitative and qualitative descriptions of these two methods, and describes the additional modifications needed to handle an arbitrary number of ac limits and dc links. A detailed account of the implementation of these methods in $\mathrm{C}$ code and the characteristics of the resulting program are also presented. This tool is then used for determining voltage profiles and loadability margins for several ac/dc systems.

\section{THE POINT OF COLLAPSE METHOD}

A general one-parameter nonlineat. dynamic system can be represented by the vector field

$$
\dot{\mathbf{z}}=\mathbf{f}(\mathrm{z}, \lambda)
$$

where $\mathrm{z} \in \mathbf{R}^{n}$ and $\lambda \in \mathbf{R}$. This system presents a bifurcation at the equilibrium point $\left(\mathrm{z}_{0}, \lambda_{0}\right)$ when the corresponding linearization (Jacobian) is singular. Saddle-node bifurcations are typical in practice $[1,10,11]$, and are characterized by the steady state Jacobian $D_{z} f\left(z_{0}, \lambda_{0}\right)$ having a simple and unique zero eigenvalue, with nonzero right eigenvector $\mathbf{v}$ and left eigenvector $\mathbf{w}$. This condition can be summarized by the set of vector equations (2) for the right eigenvector, and (3) for the left eigenvector.

$$
\begin{aligned}
\mathbf{f}(\mathbf{z}, \lambda) & =\mathbf{0} \\
D_{z} \mathbf{f}(\mathbf{z}, \lambda) \mathbf{v} & =\mathbf{0} \\
\|\mathbf{v}\| & \neq 0 \\
\mathbf{f}(\mathbf{z}, \lambda) & =\mathbf{0} \\
D_{z}^{T} \mathbf{f}(\mathbf{z}, \lambda) \mathbf{w}=\mathbf{0} & \\
\|\mathbf{w}\| & \neq 0
\end{aligned}
$$

The solution to these equations yields the saddle-node bifurcation point.

This paper tests this concept using the ordinary power flow equations for $f$. The bifurcations that are compute for the load flow equations can be directly related to bifurcations of certain dynamic equations of the form (1) as shown 
in [12]. These dynamic equations include generator swing dynamics, which depend only on frequency and the real and reactive power balance at the load. Some dynamic power system models (particularly those with detailed generator models) do not have this form. In these cases, the use of the standard load flow equations is not appropriate (see [13] and [14]). However, our approach can be extended without difficulty if the right hand side of the dynamic equations is substituted for the load flow equations. The paper also recognizes that some features of load modeling are crucial in computing voltage collapse points (see for example [15]). Attention is restricted to loads whose incremental behavior is that of classic constant PQ models. Once again, the approach extends easily to encompass changes in the load models. Load models appropriate to voltage collapse computations remain controversial.

For power system dynamic models, the parameter $\lambda$ typically stands for load increase throughout the network. One can solve equations (2) or (3) to determine the maximum loading factor $\left(\lambda_{0}\right)$ and the point of voltage collapse $\left(z_{0}\right)$. Equations (2) are used by Seydel in [1] to determine saddlenode bifurcations of general dynamic systems, and have been applied to voltage stability analysis of ac systems in $[2,3,5]$. A variation of equations (3) was used in [4] to determine voltage collapse points of purely reactive ac systems; here (as in [16]) the nonzero condition for the left eigenvector, $\|w\| \neq 0$, is replaced by the saddle-node bifurcation condition (4) depicted below. In all these cases the Jacobian can be shown to be nonsingular at the bifurcation point [7]. This makes these methods numerically appealing.

$$
\left.\mathrm{w}^{T} \frac{\partial \mathrm{f}}{\partial \lambda}\right|_{\left(z_{0}, \lambda_{0}\right)} \neq 0
$$

These PoC or direct methods have the advantage of producing right and left eigenvector information. The right eigenvector can be used to detect variables (areas) in the network prone to voltage collapse [12], whereas the left eigenvector can be used to compute an optimal control strategy to avoid saddle-node bifurcations [17]. The use of right eigenvector information to improve the maximum loadability margin for a 173 bus ac/dc system is illustrated later.

When these methods are applied to real sized ac/dc systems, there are several factors that have to be taken into account in order to obtain consistent and reliable results. Good initial guesses for the system variables, particularly the eigenvectors, are essential, otherwise a Newton-Raphson approach for obtaining the solution to the PoC equations either yields undesirable results or does not converge. This becomes important for large systems with an arbitrary number of ac/dc state variable limits.

$\mathrm{AC}$ limits considered in this paper are voltage regulating transformer and phase shifter tap limits, and voltage and reactive power limits at generator buses. The HVDC system introduces additional limits: commutation transformer tap limits, converter angle limits, and current and power limits. Enforcing ac system limits does not present major difficulties if all system variables, including reactive power at PV generator buses, are explicitly represented in the power flow equations. However, when a variable reaches an operational limit in the HVDC link, the system changes control mode, which can be modeled by a change in the state variables used to simulate the dc steady state behavior. A scheme

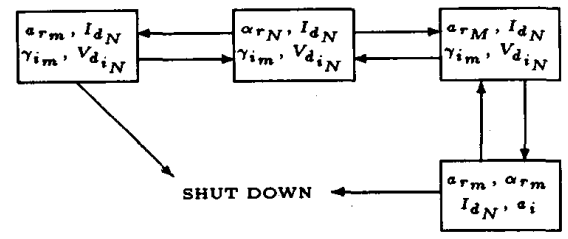

Fig. 1: Control modes of HVDC link for power flow solution, when ac bus voltage at the rectifier side changes. Margin switching at the inverter side is assumed.

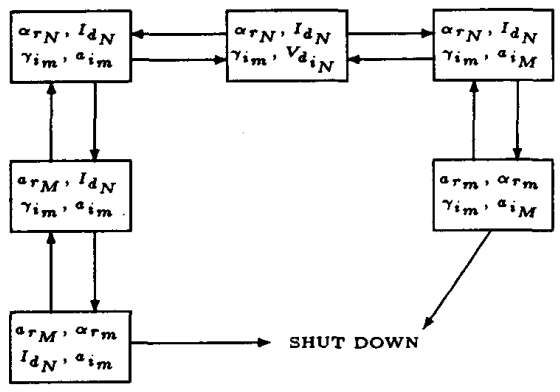

Fig. 2: Control modes of HVDC link for power flow solution, when ac bus voltage at the inverter side changes. Margin switching at the inverter side is assumed.

has to be devised to accurately represent the dynamic control logic at both converter stations. Figures 1 and 2 depict the transition logic used to simulate the switching between different control modes for the rectifier and inverter when the ac bus voltage at either side of the dc line changes. The boxes represent the four dc active control variables.

It is also possible to control, for example, $Q$ at the inverter instead of $\gamma$, or to control $P$ at the rectifier instead of $I_{d}$. In both cases, the control is performed by means of an outer loop. The control of $Q$, in particular, has the advantage of helping regulate the ac voltage in the vicinity of the inverter. However, as the system approaches an extreme point it is more than likely that both the inverter tap position $a_{i}$ and the extinction angle $\gamma_{i}$ will be pushed to their limits to provide the maximum amount of reactive support possible. This results in switching out of constant $Q$ mode and into constant $\gamma$ mode, as shown in figure 2 . Thus, whether reactive power control is represented or not, the collapse point limits found are likely to be identical. For power control, substitute active power for dc current in both figures. The notation in these figures is as follows: $r$ stands for rectifier and $i$ for inverter; $\alpha$ is the firing angle and $\gamma$ is the extinction angle; $I_{d}$ is the dc link current and $V_{d}$ is the corresponding dc voltage; $N$ represents a nominal value, $M$ is a maximum value, and $m$ is a minimum value. The reader is referred to $[18,19]$ for more details.

The state variables $z$ are initialized to the values obtained from a base case power flow solution. Initial guesses for the eigenvectors $v$ and $w$ are obtained from 4 or 5 iterations of the inverse power method [20] applied to the initial power flow Jacobian. The parameter $\lambda$ is initially set to 
zero. These initial guesses are unreliable when limits are reached. To improve the convergence characteristics of the method, new values for the eigenvectors are calculated every time the ac/dc system reaches a limit. This is done by applying a few iterations of the inverse power method to the Jacobian of the new set of ac/dc equations evaluated at the switching point.

For systems far from the bifurcation point, the initial guesses described above are not sufficient to obtain consistent results. By initially stressing the system beyond the base case one can resolve this difficulty. This initial load can be calculated using the tangent vector to the system branch $[1,10]$ at the base case (see figure 3 ), which is a technique used in continuation methods to find system voltage profiles $[5,8,9]$. This tangent vector, $\mathrm{dz} / \mathrm{d} \lambda$, can be found by a factorization and a repeat solution of the base case power flow Jacobian, $D_{z} \mathbf{f}\left(\mathbf{z}_{1}, 0\right)=\left.\frac{\partial \mathbf{f}}{\partial \mathbf{z}}\right|_{1}$, assuming a linear loading pattern. Thus,

$$
\begin{aligned}
\mathbf{f}(\mathbf{z}, \lambda)=\mathbf{0} \Rightarrow & \frac{\mathrm{d} \mathbf{f}}{\mathrm{d} \lambda}\left(\mathbf{z}_{1}, 0\right)=\left.\frac{\partial \mathbf{f}}{\partial \mathrm{z}}\right|_{1} \frac{\mathrm{d} \mathbf{z}}{\mathrm{d} \lambda}+\left.\frac{\partial \mathbf{f}}{\partial \lambda}\right|_{1}=\mathbf{0} \\
& \frac{\mathrm{d} \mathbf{z}}{\mathrm{d} \lambda}=-D_{z}^{-1} \mathbf{f}\left(\mathbf{z}_{1}, 0\right) \frac{\partial \mathbf{f}}{\partial \lambda}
\end{aligned}
$$

The amount of added load comes from the normalization of the tangent vector, i.e.,

$$
\begin{aligned}
\Delta \lambda & =\frac{k}{\|\mathrm{dz} / \mathrm{d} \lambda\|_{1}} \\
\Delta \mathrm{z} & =\Delta \lambda \frac{\mathrm{dz}}{\mathrm{d} \lambda}
\end{aligned}
$$

Equation (7) is used to calculate an initial guess $\left(\mathbf{z}_{1}+\Delta \mathbf{z}\right)$ for solving the power flow problem at the new load setting. Tests demonstrate that an accurate solution to this new power flow is not required to obtain a set of good initial values for the $\mathrm{PoC}$ variables.

The scaling constant $k$ in equations (6) denotes relative system loading. When $k=0$, the system is at base case conditions. As mentioned above, it is usually better to start the solution for some $k>0$. The initial choice of $k$ affects the performance of the method. This choice does not affect the final result. An a-priori optimal choice for initial $k$ for all cases simply does not exist. However, experiments with a variety of practical systems suggests that the degree of loadability of a system beyond a "normal" base case increases somewhat less than linearly with the number of generators in the system. That is, the more generators a system has available for dispatch, the greater the loadability. The following entirely empirical initial choice for $k$ gave good results in most of our experiments and permitted reliable "hands-off" solutions:

$$
k=\sqrt{n_{g}}
$$

where $n_{g}$ is the number of generators. If some of the generators in the system are at their limit in the base case, this number must be reduced. Several variants of this idea were tested.

Experience with the PoC method has demonstrated that the left eigenvector equations yield better results than their right eigenvector counterpart. Using an infinite norm condition as the third PoC equation in (3), i.e., $\|w\|_{\infty}=$ $\max \left\{w_{i}\right\}=1$, proves to be a more reliable way for finding the voltage collapse point.

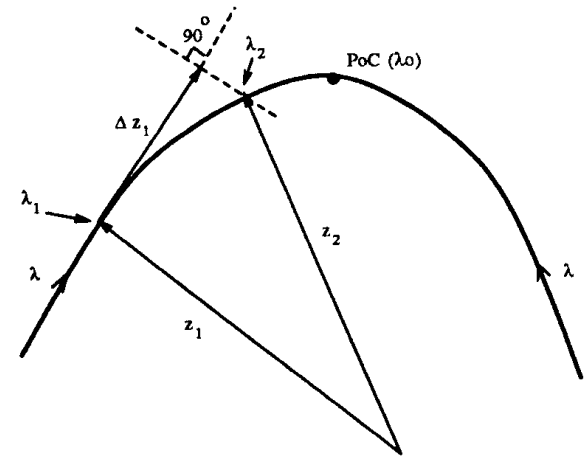

Fig. 3: Continuation method geometry in state space and parameter space.

\section{CONTINUATION METHODS}

Continuation methods present another way of determining proximity to saddle-node bifurcations in dynamic systems. These methods are thoroughly described in [1] for general systems, and were applied to the analysis of voltage collapse in ac only systems in [5, 8, 9]. References [5, 9] show a direct application of the parameterized continuation methods to the ac power flow equations. In [8], although the authors do not use the parameterization approach, a perpendicular intersection is utilized to improve the convergence characteristics of these methods. The software presented in this paper uses both techniques, i.e., parameterization and perpendicular intersection, to trace the branch (voltage profiles) of realistic ac/dc networks.

Continuation methods consist of a three step approach to tracing the equilibrium points as one parameter in the system changes, i.e., find the solutions to the power flow equations $f(z, \lambda)=0$ for a given set of parameter values. Normally the loading factor $\lambda$ is the varying parameter; however, as the system gets closer to bifurcation the classical power flow Jacobian becomes ill-conditioned. A parameterization (e.g., switching from $\lambda$ to, for example, a bus voltage $\left.z_{i} \in \mathrm{z}\right)$ makes the power flow Jacobian nonsingular at the voltage collapse point.

Figure 3 shows graphically how these methods work. The manifold, depicted as a boldface curve, represents the system equilibria as the system parameter changes. Assuming that the system is initially at the state $\left(\mathrm{z}_{1}, \lambda_{1}\right)$, one can predict the new equilibria $\left(z_{2}, \lambda_{2}\right)$ by using $\Delta \lambda$ and the scaled tangent vector $\Delta \mathbf{z}_{1}$ defined in equations $(6)$ and (7), respectively, since for a small step $z_{2} \approx z_{1}+\Delta z_{1}$ and $\lambda_{2} \approx \lambda_{1}+\Delta \lambda$. To obtain the actual values of $z_{2}$ and $\lambda_{2}$, one can use the perpendicular hyperplane to the tangent vector to find the desired point in the branch. Mathematically this can be summarized in the following three steps:

1. Predictor: Find the step $\Delta \hat{\mathrm{z}}$ and $\Delta p$ in state space and parameter space by solving equation (8).

$$
D_{\dot{z}} \mathbf{f}\left(\hat{\mathbf{z}}_{1}, p_{1}\right) \frac{\mathrm{d} \hat{\mathbf{z}}}{\mathrm{d} p}=-\left.\frac{\partial \mathbf{f}}{\partial p}\right|_{1}
$$

Hence, one can arbitrarily chose the length of the step 
to take, i.e.,

$$
\begin{aligned}
\Delta p & =\frac{k}{\|\mathrm{~d} \hat{\mathbf{z}} / \mathrm{d} p\|} \\
\Delta \hat{\mathbf{z}} & =\Delta p \frac{\mathrm{d} \hat{\mathbf{z}}}{\mathrm{d} p}
\end{aligned}
$$

The parameter $p$ is initially set to $\lambda$, and the state variables $\hat{\mathbf{z}}$ are equivalent to $z$. As the process approaches the bifurcation, $p$ is likely to change to one of the ac bus voltages (see step 3 ), with the loading factor $\lambda$ becoming part of $\hat{z}$. Although the constant $k$ is user defined, $k=1$ yields good results.

2. Corrector: Find the intersection between the perpendicular plane to the tangent vector and the branch, i.e., solve equations

$$
\begin{aligned}
& \mathbf{f}(\hat{\mathbf{z}}, p)=0 \\
& \Delta p\left(p-p_{1}-\Delta p\right)+\Delta \hat{\mathbf{z}}^{T}\left(\hat{\mathbf{z}}-\hat{\mathbf{z}}_{1}-\Delta \hat{\mathbf{z}}\right)=0
\end{aligned}
$$

where $p_{1}$ and $\hat{\mathbf{z}}_{1}$ come from the previous iteration. By initially setting $\hat{z}$ to $\hat{\mathbf{z}}_{1}+\Delta \hat{\mathbf{z}}$ and $p$ to $p_{1}+\Delta p$, solving this set of equations usually takes one or two iterations. If the process fails to converge, the steps $\Delta \hat{\mathbf{z}}$ and $\Delta p$ are cut in half until convergence is attained. Enforcing ac and $\mathrm{dc}$ limits is done with ease, since the initial guesses are good estimates of the actual solution. If the initial values violate $\mathrm{ac} / \mathrm{dc}$ limits, the steps are cut so that all limits are met, and then the continuation equations (9) are solved. When $|\Delta p|$ is cut to less than certain tolerance (typically $10^{-4}$ ), the corresponding equations are changed according to the variable limits that have been reached.

3. Parameterization: Check the relative change in all the system variables, and trade $p$ with the variable that presents the largest change. In other words,

$$
p \leftarrow \max \left\{\left|\frac{\Delta \hat{z}_{1}}{\hat{z}_{1}}\right|,\left|\frac{\Delta \hat{z}_{2}}{\hat{z}_{2}}\right|, \cdots,\left|\frac{\Delta \hat{z}_{n}}{\hat{z}_{n}}\right|,\left|\frac{\Delta p}{p}\right|\right\}
$$

Experience with the method has demonstrated that as the process approaches the bifurcation, $p$ changes from $\lambda$ to the system bus voltage that is varying the most, and after a few iterations of the method it returns back to $\lambda$. However, in all the cases tested, one can obtain good results even without switching parameters. Using this automated approach for the choice of the parameter $p$, resulted in no difficulties even in highly compensated systems.

By changing the parameter $p$ from $\lambda$ to a state variable $z_{i} \in \mathrm{z}$, one guarantees that the Jacobian of equations (8) is nonsingular at the bifurcation point [1]. At the bifurcation point the tangent vector $d z / d \lambda$ is a scaled version of the right eigenvector $v$. It can be shown that the Jacobian of equations (9) is also nonsingular at the voltage collapse point, even for $p=\lambda$ (singular power flow Jacobian). Notice that approximate right eigenvector information can be obtained from this method when close to the point of voltage collapse.

The method naturally goes around the collapse point, allowing the user to trace the "unstable" side of the branch.

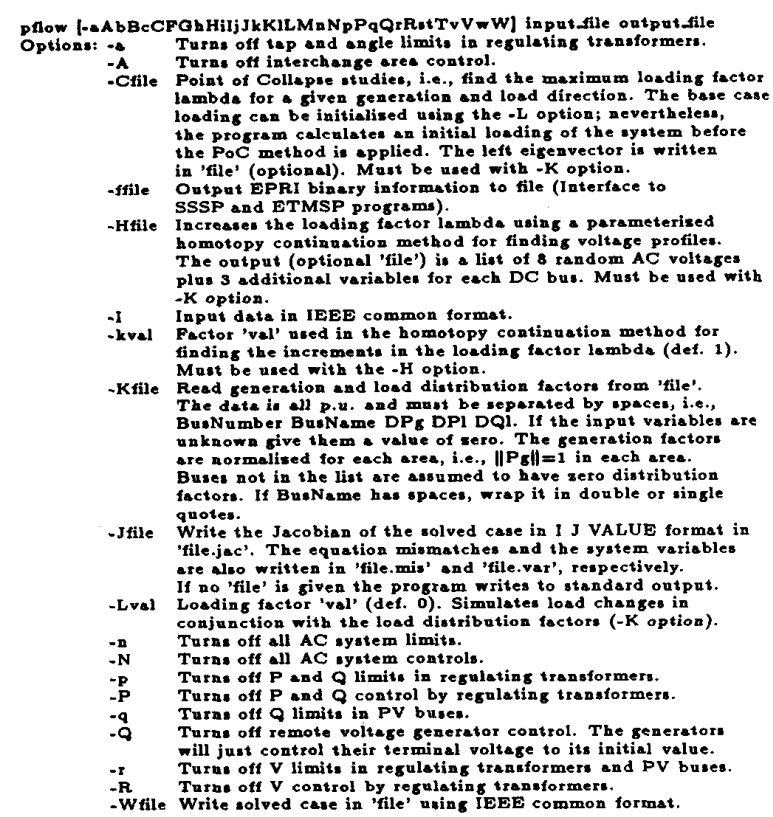

Fig. 4: Part of the PoC power flow help feature.

This turning point must be detected in order to change the sign of $\Delta p$ in equations ( 8 ). For $p=\lambda$, the bifurcation point can be detected by a sign change in the determinant of the power flow Jacobian, which is a by-product of its factorization. On the other hand, for $p=z_{i}$, the turning point is detected by a sign change in $\Delta \lambda$.

\section{THE POINT OF COLLAPSE POWER FLOW}

This program is a portable $\mathrm{C}$ code implementation of the methods described above. It has been successfully tested in several Unix workstations, namely, SUN, HP and DELL, and is also running in PC machines under DOS 3.3 (a Windows 3.0 version is currently under development). The program is capable of handling any size system, limited only by the available memory and swap space. It reads WSCC [21], EPRI [22], and Common Format [23] input files, and produces a variety of ASCII and binary files, depending on the user defined options. It was designed to be a production type software for research and commercial applications, hence, speed and versatility were the main concerns during the development of this program.

The program solves the ac/dc power flow using a NewtonRaphson iterative process with automatic step-size adjustment. The factorization routines were taken from the Sparse Matrix Manipulation System (SMMS) [24]. The PoC and continuation methods were implemented with all the practical details explained in the two previous sections. A solution to the voltage collapse problem can be obtained for any ac/dc systems with a variety of operational limits.

To test the validity and performance of the program, results were compared against three commercial software 
packages: EPRI's power flow versions 3 and 5 [21, 22], and Electrocon's power flow version 3.10 [25]. The results and performance yielded by this program are shown and discussed in the next section.

Figure 4 shows some of the user defined options of the PoC power flow. Notice that the load distribution factors (DPl, DQl) and the generator participation factors (DPg) defined using the $-\mathrm{K}$ option, give a direction in demand space and generation space to realistically solve the voltage collapse problem. However, these directions can also be used to solve the base case power flow problem with a distributed slack-bus and different loading levels.

\section{RESULTS}

The program was tested in a variety of ac/dc systems, ranging from 14 to 2158 buses. Figure 5 depicts a 14 bus system designed to replicate some of the characteristics of electric networks in the western part of the United States. The 173 bus network is a reduced version of a real 2158 bus system developed jointly with CEPEL. A 133 bus system, which is a modification of the 173 bus system, was also used.

Figures 6 and 7 show two different set of voltage profiles obtained by applying the continuation method to the 173 and $2158 \mathrm{ac} / \mathrm{dc}$ bus systems, respectively. The first profile shows the sharp voltage changes due to the reactive power limits at the generation buses. This is an issue reported in [4], and is produced by the loss of reactive support throughout the network when a generator or group of generators reach a $Q$ limit, hence, sharply reducing the maximum loadability margin $\lambda_{0}$ of the system. For example, $\lambda_{0}$ for the 173 bus system without $Q$-limits is $6.0725 \%$ above the initial system load, whereas when these limits are included $\lambda_{0}=2.0275 \%$.

Figure 7 shows another interesting phenomenon where the system becomes immediately unstable when a generator reaches a reactive limit. This was also observed in the 133 bus system. This seems to occur rather close to the bifurcation. (For a detailed explanation of this kind of instability the interested reader is referred to the work by Dobson et al in [26].)

The "nose" of the curves in Figure 7 requires some explanation. When obtaining these curves it is assumed that $P V$ (generation) buses are operated at constant $V$ until the limit for $Q$ is reached, and only then is the value of $Q$ fixed at its limit. For these curves, the system does not, strictly speaking, reach a bifurcation based on this operating regime. This is because the point of maximum loadability occurs at the point where a $Q$ limit is reached. If the Point of Collapse method is applied to the equations considering this limit, no solution possible and the method fails. However, if one were to permit $Q$ to reach its limit, and then ask the question of where is the nearest bifurcation point with $Q$ fixed at this limit, then one would discover that this bifurcation point occurs at a slightly higher voltage, and that this point allows a slightly larger power transfer capability than the solution based on holding the voltage constant until the $Q$ limit is reached. This is the meaning of the incomplete upper "third solution" near the nose. The point is not entirely fictitious: a different operating policy for the system, based on extra $Q$ injection at the bus, rather than constant $V$, would permit this point to be reached. However, in every case tested the difference in the loadability level attainable with either method was virtually negligible.

It was confirmed that constant active power control in the HVDC link, as opposed to constant dc current control, reduces the loadability margin of the ac/dc system [27, 28]. For the 14 bus system, changing the HVDC control mode from constant power to constant current in both dc links, increases $\lambda_{0}$ from $11.269 \%$ to $11.568 \%$.

Another important feature of the $\mathrm{PoC}$ method is that it yields the right or left eigenvectors corresponding to the zero eigenvalue at the bifurcation point. For the 173 bus system, the maximum element values of the saddle-node right eigenvector shows that a lack of reactive power support in the area conformed by buses 74,75 and 76 is the source of the voltage stability problem. Hence, increasing the shunt reactive support from the original $550 \mathrm{MVAR}$ to $850 \mathrm{MVAR}$ in bus 75 , moves the collapse point from $\lambda_{0}=2.0275 \%$ to $\lambda_{0}=2.3117 \%$. (For examples on possible applications of the left eigenvector see [17].)

Table 1 shows the maximum loading factors $\lambda_{0}$, and the time performance for different $\mathrm{ac} / \mathrm{dc}$ and ac systems obtained in a SUN-SPARC IPC Unix workstation. The times shown include all program operations, including input and output, which in a dedicated Unix workstation correspond to real times. The ac only systems in this table were simulated by treating the HVDC links as constant active and reactive power injections. For the 2158 bus system, several types of regulating transformers, area interchange power control, bus voltage and reactive limits are included. Limits significantly increase the computation time.

The convergence problems observed in the last two entries of table 1 when using the continuation method are due to the sharp turning point and the step cutting technique used. The predictor, when applied to a "sharp" nose curve close to the bifurcation, yields a large step. Thie creates convergence problems for the corrector part of the method (indicated by $\ddagger$ in the table), since there is no crossing with the bifurcation branch of the equilibria. Step cutting would produce the right answer; however, if the step is cut below the convergence tolerance of the Newton solver, the program will take this slightly incorrect value as the solution.

The PoC method consistently performed faster, by about a factor of 2, than the continuation method. This method also generates right and left eigenvector information that can be used to increase loadability margins. However, continuation methods have the advantage of producing voltage profiles, and as a by-product yield unstable equilibrium points that can be useful for system stability analysis. Another advantage of the continuation methods is that they are able to detect immediate instabilities due to reactive power limits, although in practice these points and the bifurcations are very close. Finally, the step cutting technique used in the continuation methods to improve convergence can lead to slightly incorrect results in cases of sharp turning points.

\section{CONCLUSIONS}

A detailed description of the implementation of the $\mathrm{PoC}$ and continuation methods is presented, including the practical considerations that allow to obtain consistent results in a diversity of ac and ac/dc systems. A brief description of 


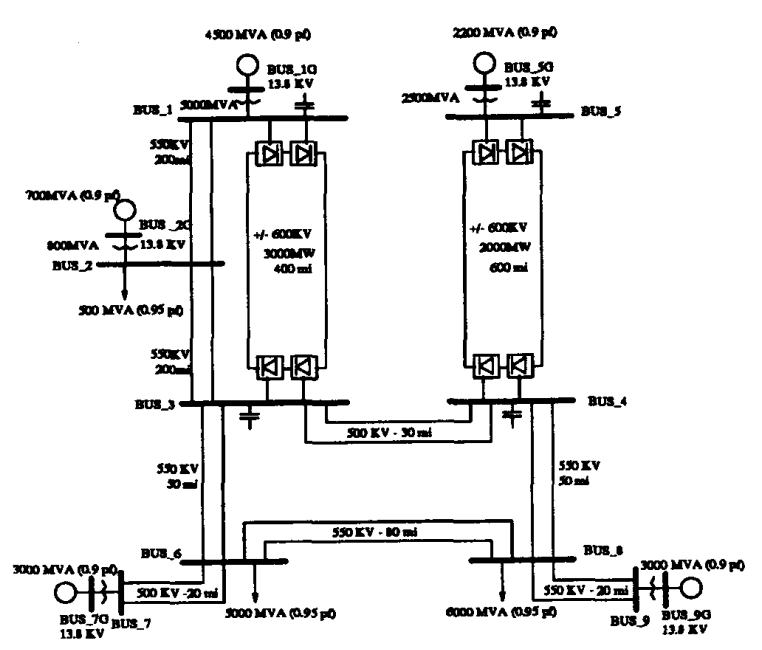

Fig. 5: 14 bus ac/dc system.

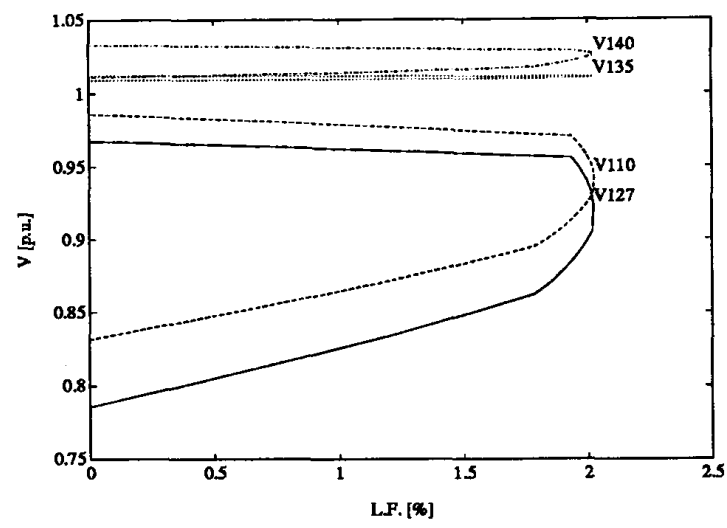

Fig. 6: Voltage Profiles for 173 bus ac/dc system. Sharp corners correspond to generator $Q$ limits.

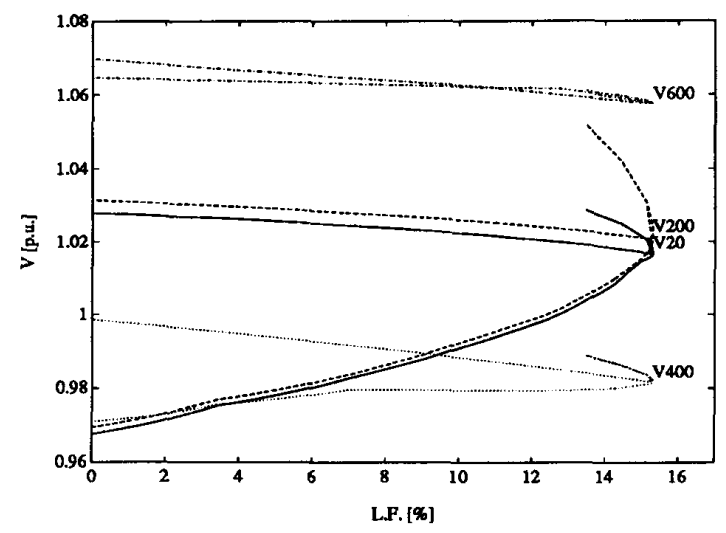

Fig. 7: Voltage Profiles for 2158 bus ac/dc system. Corner points correspond to Q-limit instabilities. The PoC is only slightly beyond.

\begin{tabular}{|c|c|c|c|c|c|}
\hline \multirow[t]{2}{*}{ System } & \multirow{2}{*}{$\begin{array}{l}\text { P.F. } \\
\text { time }\end{array}$} & \multicolumn{2}{|c|}{ Continuation } & \multicolumn{2}{|c|}{$\mathrm{POC}$} \\
\hline & & $\lambda_{0}[\%]$ & time & $\lambda_{0}[\%]$ & time \\
\hline $\begin{array}{l}14 \text { AC buses } \\
2 \text { DC bipoles } \\
21 \text { AC elem. } \\
5 \text { Gen. } \\
2 \text { Areas }\end{array}$ & $\overline{0.48}$ & 11.567 & $7.9 \mathrm{~s}$ & 11.5683 & 3.68 \\
\hline $\begin{array}{l}173 \mathrm{AC} \text { buses } \\
255 \mathrm{AC} \text { elem. } \\
29 \mathrm{Gen} .\end{array}$ & 6.58 & 2.7591 & 43.38 & 2.76086 & $25.4 \mathrm{~s}$ \\
\hline $\begin{array}{l}17 \overline{3} \text { AC buses } \\
2 \text { DC bipoles } \\
255 \text { AC elem. } \\
29 \text { Gen. }\end{array}$ & $5.7 \mathrm{~s}$ & $2 . \overline{0254}$ & $44.1 \mathrm{~s}$ & 2.0275 & $23.3 \mathrm{~s}$ \\
\hline $\begin{array}{l}133 \mathrm{AC} \text { buses } \\
213 \mathrm{AC} \text { elem. } \\
41 \mathrm{Gen} \text {. }\end{array}$ & $\overline{4.3 \mathrm{~s}}$ & $4.9983^{\dagger}$ & 45.58 & 4.99949 & $29.9 \mathrm{~s}$ \\
\hline $\begin{array}{l}133 \mathrm{AC} \text { buses } \\
2 \mathrm{DC} \text { bipoles } \\
213 \mathrm{AC} \text { elem. } \\
41 \mathrm{Gen} .\end{array}$ & 5.18 & $5.0021^{\dagger}$ & $52.1 \mathrm{~s}$ & 5.00339 & $34.9 \mathrm{~s}$ \\
\hline $\begin{array}{l}2158 \text { AC buses } \\
2 \text { DC bipoles } \\
3402 \text { AC elem. } \\
384 \text { Gen. } \\
62 \text { V reg. trf. } \\
8 \text { PQ reg. trf. } \\
23 \text { Areas }\end{array}$ & $10.3 \mathrm{~m}$ & $15.275^{\dagger}$ & $\begin{array}{c}5 \mathrm{~h} \\
11.0 \mathrm{~m}\end{array}$ & 15.2986 & $\begin{array}{c}1 \mathrm{~h} \\
31.2 \mathrm{~m}\end{array}$ \\
\hline $\begin{array}{l}2158 \text { AC buses } \\
2 \text { DC bipoles } \\
3402 \text { AC elem. } \\
384 \text { Gen. }\end{array}$ & $2.8 \mathrm{~m}$ & $32.688^{f}$ & $53.4 \mathrm{~m}$ & 32.6799 & $38.4 \mathrm{~m}$ \\
\hline $\begin{array}{l}2158 \text { AC buses } \\
3402 \text { AC elem. } \\
384 \text { Gen. }\end{array}$ & $2.8 \mathrm{~m}$ & $32.686^{\ddagger}$ & $\begin{array}{c}1 \mathrm{~h} \\
5.5 \mathrm{~m}\end{array}$ & 32.6786 & $29.5 \mathrm{~m}$ \\
\hline
\end{tabular}

Table 1: Continuation and PoC method results for SUN SPARC-IPC Unix workstation.

the computational characteristics and requirements of these methods is also shown. Finally, some of the test cases and results are discused, in particular those related to a real ac/dc 2158 bus network with a variety of operational limits and controls.

The PoC method yields voltage sensitivity information and time performances that justify its use as a production tool. The performance of this method can be significantly enhanced by using specialized sparse matrix techniques to reduce computation times, like block factorization as suggested in [4]. On the other hand, continuation methods, although slower, produce additional information that can be used in the analysis of other stability issues. For these two reasons the authors believe that these two methods complement each other, particularly for systems where one of the methods might fail in producing the desired results.

\section{ACKNOWLEDGEMENTS}

The support of this work by the Electric Power Research Institute, project RP 2675-4 under the direction of Stig Nilsson, is gratefully acknowledged. Dr. Cañizares also received support from Fulbright, OAS and EPN. Both authors also thank Professor Ian Dobson for his valuable comments.

\section{REFERENCES}

[1] R. Seydel, From Equilibrium to Chaos-Practical Bifurcation and Stability Analysis, Elsevier Science Publishers, North-Holland, 1988. 
[2] F. L. Alvarado and T. H. Jung, "Direct Detection of Voltage Collapse Conditions," Proceedings: Bulk Power System Voltage Phenomena - Voltage Stability and Security, EPRI EL-6183, Jan. 1989, pp. 5.23-5.38.

[3] V. Ajjarapu, "Identification of Steady-State Voltage Stability in Power Systems," IASTED Proceedings of International Conference on High Technology in the Power Industry, Phoenix, Arizona, March 1988, pp. 244-247.

[4] T. Van Cutsem, "A Method to Compute Reactive Power Margins with respect to Voltage Collapse," IEEE Trans. Power Systems, Vol. 6, No. 2, Feb. 1991, pp. 145-156.

[5] H. D. Chiang, W. Ma, R. J. Thomas, J. S. Thorp, "A Tool for Analyzing Voltage Collapse in Electric Power Systems," Proceedings of the Tenth Power System Computation Conference, Graz, Austria, August 1990, pp. 1210-1217.

[6] C. A. Cañizares, F. L. Alvarado, C. L. DeMarco, I. Dobson, W. F. Long, "Point of Collapse Methods Applied to AC/DC Power Systems," IEEE/PES 91 SM 491-1 PWRS, July 1991.

[7] C. A. Cañizares, F. L. Alvarado, C. L. DeMarco, I. Dobson, W. F. Long, "Voltage Collapse and Transient Energy Function Analyses of AC/DC Systems," Report ECE-90-7, University of Wisconsin-Madison, Nov. 1990.

[8] K. Iba, H. Suzuki, M. Egawa, T. Watanabe, "Calculation of Critical Loading Condition with Nose Curve Using Homotopy Continuation Method," IEEE/PES 90 SM 415-0 PWRS, July 1990.

[9] V. Ajjarapu and C. Christy, "The Continuation Power Flow: A Tool for Steady State Voltage Stability Analysis," IEEE PICA Conference Proceedings, May 1991, pp. 304-311.

[10] J. Guckenheimer and P. Holmes, Nonlinear Oscillations, Dynamical Systems, and Bifurcations of Vector Fields, Springer-Verlag, New York, USA, 1986.

[11] I. Dobson and H. D. Chiang, "Towards a Theory of Voltage Collapse in Electric Power Systems," Systems \& Control Letters 13, 1989, pp. 253-262.

[12] I. Dobson, "Observations on the Geometry of Saddle Node Bifurcations and Voltage Collapse in Electric Power Systems," to appear in IEEE Trans. on Circuits and Systems, Part 1, scheduled for March 1992.

[13] C. Rajagopalan, B. Lesieutre, P. W. Sauer and M. A. Pai, "Dynamic Aspect of voltage/Power Characteristics," IEEE/PES 91 SM 419-2 PWRS, San Diego, CA, July 28August 11991.

[14] P. W. Sauer and M. A Pai, "Power System Steady State Stability and the Load-Flow Jacobian," IEEE Trans. Power Systems, Vol. 5, No. 4, November 1990, pp. 1333-1344.

[15] G. Gao, G. K. Morrison and P. Kundur, "Voltage Stability Evaluation Using Modal Analysis," IEEE/PES 91 SM 420-0 $P W R S$, San Diego, CA, July 28-August 11991.

[16] I. Dobson, L. Lu and Y. Hu, "A Direct Method for Computing a Closest Saddle Node Bifurcation in the Load Parameter Space of an Electric Power System", Proceedings of the International Symposium on Circuits and Ssytems, Singapore, June 1991, pp. 3019-3022.

[17] I. Dobson and L. Lu, "Computing an Optimal Direction in Control Space to Avoid Saddle Node Bifurcation and Voltage Collapse in Electric Power Systems," to appear in IEEE Transaction on Automatic Control, scheduled for October 1992.

[18] J. Arrillaga, High Voltage Direct Current Transmission, Peter Peregrinus Ltd., London, UK, 1983.

[19] J. Arrillaga, C. P. Arnold, B. J. Harker, Computer Modelling of Electrical Power Systems, John Wiley \& Sons, UK, 1983.
[20] S. D. Conte and Carl de Boor, Elementary Numerical Analysis - An Algorithmic Approach, Third Edition, McGrawHill, New York, USA, 1980.

[21] Extended Transient-Midterm Stability Package: User's Manual for the Power Flow Program, EPRI EL-2002-CCM, January 1987.

[22] Methodology for the Integration of HVDC Links in Large AC Systems - Phase 2: Advanced Concepts, EPRI EL4365-CCM, Vol. 3, April 1987.

[23] IEEE Committee Report, "Common Format for Exchange of Solved Load Flow Data," IEEE Trans. Power Apparatus and Systems, Vol. PAS-92, No. 6, Nov./Dec. 1973, pp. 19161925.

[24] F. L. Alvarado, "Manipulation and Visualization of Sparse Matrices," ORSA Journal of Computing 2, 1990, pp. 180207.

[25] TRANSMISSION 2000 Power Flow Program User's Reference Manual, Electrocon International Inc., Ann Arbor, Michigan, July 1989.

[26] I. Dobson and L. Lu, "Immediate Change in Stability and Voltage Collapse when Generator Reactive Power Limits are Encountered," Bulk Power System Voltage Phenomena, Voltage Stability and Security NSF Workshop, Deep Creek Lake, Maryland, August 4-7, 1991.

[27] B. Frankén and G. Anderson, "Analysis of HVDC converters connected to Weak AC Systems," IEEE Trans. Power Systems, Vol. 5, No. 1, Feb. 1990, pp. 235-242.

[28] L. A. S. Pilotto, M. Szechtman, A. E. Hammad, "Transient AC Voltage Related Phenomena for HVDC Schemes Connected to Weak AC Systems," IEEE/PES 91 SM 305-3. $P W R D$, July 1991.

Claudio A. Cañizares (S'87) was born in Mexico, D.F. in 1960. In April 1984, he received the Electrical Engineer degree from the Escuela Politécnica Nacional (EPN), Quito-Ecuador, where he is currently an Associate Professor. In 1991 he obtained the $\mathrm{Ph}$.D. degree from the University of Wisconsin-Madison. Mr. Cañizares is currently finishing his $\mathrm{PhD}$ studies at the University of Wisconsin-Madison, sponsored by Fulbright, OAS, and EPN.

Fernando L. Alvarado (M'69, SM'78) was born in Lima, Peru. He received the $B E E$ and $P E$ degrees from the National University of Engineering in Lima, Peru, the MS degree from Clarkson University, and the $\mathrm{PhD}$ degree from the University of Michigan in 1972. Since 1975 he has been with the University of Wisconsin in Madison, where he is a Professor of Electrical and Computer Engineering. 


\section{Discussion}

M. K. Pal (Public Service Electric and Gas Company, Newark, N.J.): This paper does not seem to differentiate between the loadability limit obtained from the solution of the steady-state network equations and the actual voltage stability limit (or collapse point). Contrary to what is claimed in the paper, dynamic saddle-node bifurcation, which is also equated with voltage collapse points, has never been rigorously shown to occur when the corresponding power flow Jacobian becomes singular. Actually, for the constant P, Q load assumed by the authors in their analysis, the dynamic saddle-node bifurcation will occur well before the point at which the power flow Jacobian becomes singular. This is seen from the dynamic system model of equation (1) of the paper. Since the dynamic equations include the generator swing dynamics, even with a simple generator model, the system Jacobian (obtained from equation 1) is not the same as the power flow Jacobian. The bifurcation computed from load flow equations cannot, therefore, be related to bifurcation of the dynamic equation (1). This discussor recognizes that such claims have been made in the literature. However, these have never been justified on a rigorous theoretical basis.

The loadability limit depends on the load level as well as the distribution of load throughout the system, which is rarely known precisely. As such, the determination of the exact limit based on an assumed load distribution is not very meaningful. Conventional power flow can get fairly close to the limit very easily.

We also take issue with the authors' method of handling the reactive power limit. While it is true that the loadability margin is greatly reduced by the generators reaching reactive limits, the reactive limit should not be modeled by simply changing a generator bus from a PV bus to a PQ bus. Reference [26] of the paper addresses the problem correctly, as perhaps implied by the authors in the paper.

The statement " . with $Q$ fixed at this limit bifurcation point occurs at a slightly higher voltage ..." is a little confusing. Further clarification would be appreciated. Does this mean that at nominal $\mathrm{V}$, the $\mathrm{Q}$ limit will never be reached?

C. A. Cañizares*, F. L. Alvarado** (*Escuela Politécnica NacionalQuito, **University of Wisconsin-Madison): The authors wish to thank Mr. M. K. Pal for his interest on the paper. He raises several issues that must be addressed for completeness of the paper. Most of Mr Pal's comments have been already discussed in the closure of [A1]; however, we will try to address and answer all of Mr. Pal's concerns and questions.

Although the intention of the paper is just to compare two different techniques of efficiently detecting singularity of static equations' Jacobians, we would like to comment on Mr. Pal's observation regarding whether the ordinary power flow can be used to detect actual saddlenode bifurcations of dynamic equations (1). We disagree with $\mathrm{Mr}$ Pal's assertion that singularity of power flow Jacobians have not been formally proven to correspond to true dynamic saddle-node bifurcations. Reference [A3] shows that for a simple dynamic power system model, where generator swing dynamics and ordinary load dynamics are considered, the actual power flow equations can be used to detect the real bifurcation point. This can be shown using the simple example that Mr. Pal mentions, i.e., a generator-line-PQ load system, as depicted in the closure to [A1], where it can be demonstrated that the dynamic saddle-node bifurcation, due to generator swing dynamics, corresponds to the maximum power transfer point (maximum loadability limit); this point is also the point of voltage collapse. Saddle-node bifurcation conditions for a dynamic ac/dc system model also have equivalent conditions in the corresponding ordinary power flow equations for constant. PQ load models. Theorems to this effect have appeared in [A2]. Some of these ideas have been discussed in [A1]. The form of the static load model used in important, since it can be shown that constant current and constant impedance load models yield bifurcation points that do not correspond to the maximum power transfer limit. We completely "agree" with Mr. Pal that for more complicated dynamic models the complete set of static system equations must be used to detect these kinds of bifurcations. This has also been pointed out by several other authors (e.g., [14]).

As to whether the saddle-node bifurcation point for certain loading pattern is not of real interest, to paraphrase Mr. Pal, we think that this is an oversimplification. The PoC and continuation methods not only yield the limit to collapse, which gives the user an idea on how loaded the system is, but it also yields the areas prone to voltage stability problems (right eigenvector $\mathbf{v}$ ) and how to most effectively prevent these problems (left eigenvector w). Furthermore, the left eigenvector can be used to iteratively detect the closest bifurcation point for any loading pattern [16]. We also agree that ordinary power flow programs can be used to get solutions close to these bifurcation points; however, using ordinary power flow programs instead of the proposed methods is equivalent to using trail-and-error methods to find solutions of nonlinear systems of equations instead of more efficient methods. The methods discussed in the paper are by far better than simple power flow runs, since they have better numerical properties at the singular point, yield more information, and find the saddle-node bifurcation point automatically without the need for manual user intervention.

Regarding the modeling of the generator voltage control loop by simply assuming the generator terminal voltage constant within generator Q-limits, we agree that this is not the exact way to deal with the problem from the dynamic view point. The model proposed in [26] is more adequate. Nevertheless, the VQ model is a first approximation to the real generator voltage control system, and it is a widely accepted way of handling the voltage regulator phenomena when close to equilibria [A4], which is what bifurcation analysis is all about.

Finally, the statement made in the paper about the voltage value at the bifurcation point when Q-limits are considered is just an empirical observation about the actual values obtained for different cases in several systems. This comment was made just to support the notion that $Q$-limits reduce loadability margins when constant $P Q$ load models are used.

\section{References}

[A1] C. A. Cañizares, F. L. Alvarado, C. L. DeMarco, I. Dobson, W. F. Long, "Point of Collapse Methods Applied to AC/DC Power Systems," IEEE Transactions on Power Systems, Vol. 7, No. 2, May 1992, pp. 673-683.

[A2] C. A. Cañizares, Voltage Collapse and Transient Energy Function Analysis of $A C / D C$ Systems, Doctoral Thesis, The University of Wisconsin, Madison, September 1991.

[A3] I. Dobson, "Observations on the Geometry of Saddle Node Bifurcations, and Voltage Collapse in Electrical Power Systems," IEEE Transactions on Circuits and Systems-I: Fundamental Theory and Applications, Vol. 39, No. 3, March 1992, pp. 240-243.

[A4] C.-C. Liu and K. Vu, "Types of Voltage Problems," Bulk Power System Voltage Phenomena, Voltage Stability and Security NSF Workshop, Deep Creek Lake, MD, August 4-7, 1991.

Manuscript received June 19, 1992. 